\title{
Clinical Use of the Low-Molecular-Weight Heparins in Cancer Patients: Focus on the Improved Patient Outcomes
}

\author{
Bo H. Chao, ${ }^{1,2}$ Lisa Lepeak, ${ }^{1,2}$ Ticiana Leal, ${ }^{1,2}$ and H. Ian Robins, ${ }^{1,3}$ \\ ${ }^{1}$ Department of Medicine, University of Wisconsin School of Medicine and Public Health, 600 Highland Avenue, \\ Madison WI 53792, USA \\ ${ }^{2}$ University of Wisconsin Paul Carbone Comprehensive Cancer Center, 600 Highland Avenue, Madison, WI 53792, USA \\ ${ }^{3}$ Departments of Medicine, Human Oncology, and Neurology, University of Wisconsin School of Medicine and Public Health, \\ 600 Highland Avenue, Madison, WI 53792, USA
}

Correspondence should be addressed to H. Ian Robins, hirobins@wisc.edu

Received 2 November 2010; Accepted 17 February 2011

Academic Editor: Ted Wun

Copyright (C) 2011 Bo H. Chao et al. This is an open access article distributed under the Creative Commons Attribution License, which permits unrestricted use, distribution, and reproduction in any medium, provided the original work is properly cited.

\begin{abstract}
Patients with malignant neoplastic diseases represent a high-risk population relative to thromboembolic disease. With the advent of improved and accessible diagnostic technology, for example, ultrasound and/or spiral CT scans, timely diagnosis of venous thromboembolic events (VTE) is readily accomplished. The introduction of low-molecular-weight heparin (LMWH) approximately two decades ago (in contrast to unfractionated heparin and vitamin $\mathrm{K}$ antagonists) has provided a class of agents with a favorable therapeutic index. In the review to follow, the literature regarding the use of LMWH in oncologic patient populations is summarized. Topics addressed include prophylaxis, and treatment as well as consideration of the potential antineoplastic properties of this class of drugs.
\end{abstract}

\section{Introduction}

It is well recognized that cancer patients are at risk for venous thromboembolic events (VTEs) [1] with an estimated annual incidence of VTE in the cancer population five times higher than the general population [2]. This is in part due to chronic hemostatic activation and changes, including increased platelet adhesion and disseminated intravascular coagulation, which are commonly observed in cancer patients [3-10]. Although it is difficult to compare rates across studies, several generalizations regarding VTE can be made. An estimated 15\% of all patients with cancer will develop VTE during their clinical course [11]. The risk of developing VTE is significantly higher even early in diagnosis with a reported $>50$-fold higher incidence in the first 3 months following a diagnosis of cancer in one case controlled study [12]. Patients admitted to an inpatient oncology service are reported to have rates of VTE up to $8 \%$ $[13,14]$; those who have a remote diagnosis of cancer are at a lower risk, as would be expected $[13,15,16]$. Additionally, other factors may represent more significant risk variables in cancer patients such as certain types of chemotherapy $[17,18]$.

Chemotherapy is associated with a 2- to 6-fold increased risk of VTE referenced to the general population [1719]. For instance, in a study of 205 patients with stage II breast cancer randomized to 36 weeks of chemotherapy (cyclophosphamide, methotrexate, fluorouracil, vincristine, and prednisone) or 12 weeks of chemohormonal therapy (cyclophosphamide, methotrexate, fluorouracil, vincristine, prednisone, doxorubicin, and tamoxifen), there was a $6.8 \%$ incidence of deep venous thrombosis (DVT). Of note, all of the events occurred during the chemotherapy treatment period, suggesting that chemotherapy for breast cancer contributes to thrombosis [20]. A prospective observational study by Khorana et al. in 2007 showed VTE as one of the leading causes of noncancer death among 4,466 patients receiving outpatient chemotherapy, with $9.2 \%$ of deaths secondary to VTE and one death related to pulmonary embolism in a patient without known metastatic disease [19]. This is a trend that was again demonstrated in a prospective study of 4,000 patients which showed VTE was 
an independent risk factor for mortality during the first months of chemotherapy even after adjusting for several risk factors. Cancer diagnosed at the same time or within 1 year after an episode of VTE was associated with worse prognosis and relatively low rate of survival at one year (38 percent versus 47 percent in the control group) [21].

Although specific incidence rates may vary based on the clinical setting, recent reviews have highlighted the significance of histological diagnosis $[18,22]$. Patients with cancers of the pancreas [23] and brain [24], in some series, can have rates exceeding $25 \%$; other cancers associated with the highest rates of VTE exceeding 5\% include cancers of the gastrointestinal tract, ovary, uterus, kidney, and lung [18]. More recent studies have identified VTE in association with hematologic malignancies as being comparable to solid tumor patients [22]. It is interesting to note oncogenes responsible for neoplastic transformation in leukemia also may be involved in clotting activation [22]. As is the case with solid tumors, therapy for hematologic malignancies can place patients at increased risk. For example, thalidomide $[25,26]$ and lenalidomide $[27,28]$ have been associated with high rates of VTE exceeding 25\%. In addition to malignancy as a risk factor, other interventions in cancer patients, such as surgery and the use of venous access devices, may further increase the risk for thromboembolic complications [29].

In summary, as VTE contributes to morbidity and is one of the leading causes of noncancer death among patients with cancer $[19,21,30]$, the timely diagnosis and effective treatment of VTE is of paramount importance. In the following text we will discuss the existing data for the treatment and prophylaxis of VTE in cancer patients. The review will focus on the use of low-molecular-weight heparins (LMWHs), as the published literature and experience center on this class of anticoagulants in relation to both safety and efficacy. Encompassed in this discussion will be a review of the data suggesting the potential for antineoplastic effects with the application of LMWH, and their influence on patient outcomes. Relative to this, there are in vitro and animal studies showing anticoagulants, in particular the LMWHs, have an antineoplastic effect through mechanisms such as interference with tumor cell adhesion, invasion, metastasis formation, and angiogenesis [31-42].

\section{Low-Molecular-Weight Heparin as Treatment for Venous Thromboembolism}

\subsection{Pharmacology and Pharmacokinetics of Low-Molecular-} Weight Heparin. There are various FDA approved LMWHs commercially available which are produced by chemical or enzymatic depolymerization of unfractionated heparin (UFH). The fragmented LMWHs are approximately $1 / 3$ the size of UFH and range from 4,000-6,500 Daltons with a chain length of 12-22 polysaccharides. LMWH's anticoagulant activity is similar to UFH; however, a shortened polysaccharide chain allows for more specific binding to inhibit factor Xa. Unfractionated heparin binds to antithrombin, initiating inhibition of several serine proteases in the coagulation cascade, including factor IIa and Factor Xa. The polysaccharide chain is truncated in LMWHs while retaining the high affinity pentasaccharide binding sites. This results in inhibition of factor $\mathrm{Xa}$, but the shortened polysaccharide chain is relatively ineffective at binding, resulting in loss of inhibition of factor IIa [43]. In terms of anticoagulation, this results in a reduced antifactor IIa activity relative to factor Xa and improved pharmacokinetics.

Unfractionated heparin is generally given intravenously (if used therapeutically) and requires close monitoring of activated partial thromboplastin time (APTT) with frequent dose adjustments. In contrast, LMWHs are injected subcutaneously once or twice daily and do not typically require routine monitoring. Special considerations for monitoring should be given for patients with renal failure and obesity. LMWHs are cleared by the kidneys, and dosing is weight based. The biological half-life is prolonged in patients with renal failure. Dosing in renal failure and weight adjusted dosing were not addressed in original clinical trials, and monitoring of anti-Xa levels is recommended [44, 45]. Monitoring in pregnancy may also be considered given the relative increase in renal clearance [46]. Anti-Xa levels can be monitored in serum levels 4 hours following subcutaneous administration. Various labs are available for monitoring anti-Xa levels, and the target therapeutic range is $0.6-$ $1.0 \mathrm{IU} / \mathrm{mL}[47,48]$.

2.2. Safety and Efficacy. Many consider LMWH as the agent of choice for the initial and long-term treatment of VTE in patients with neoplastic disease, based on randomized clinical studies [19, 49-52]. In 2003, Lee et al. conducted a randomized study of cancer patients with VTE: 336 patients received dalteparin at $200 \mathrm{IU} / \mathrm{kg}$ daily for 5 to 7 days followed by a vitamin $\mathrm{K}$ antagonist with target international normalized (INR) ratio of 2-3 for 6 months, whereas the other 336 patients received dalteparin at $200 \mathrm{IU} / \mathrm{kg}$ daily for 1 month followed by $150 \mathrm{IU} / \mathrm{kg}$ daily for 5 months [53]. (It should be noted that the concept of a $25 \%$ dose reduction after initial therapy was an arbitrary choice, and not evaluated in comparison to other possibilities.) In this study, the cumulative risk of symptomatic and recurrent VTE during 6 months of anticoagulation was 17\% for patients treated with a vitamin $\mathrm{K}$ antagonist, compared with $9 \%$ for patients treated with dalteparin, a LMWH [53]. The relative risk reduction of $52 \%$ was statistically significant (hazard ratio $=0.48 ; P=.002$ ). One episode of recurrent VTE was prevented for every 13 patients treated with dalteparin. In terms of side effects, no difference in major or minor bleeding was detected between the groups, with $6 \%$ of dalteparin-treated patients and $4 \%$ of control patients having major bleeding episodes [53].

In another study with a similar design, Meyer and colleagues randomized 138 patients to receive either enoxaparin at $1.5 \mathrm{mg} / \mathrm{kg}$ daily for 4 days followed by warfarin with target INR of 2-3 for 3 months, or enoxaparin at $1.5 \mathrm{mg} / \mathrm{kg}$ daily for 3 months [50]. There was a consistent but not statistically significant benefit with enoxaparin over warfarin, in terms of decreased VTE recurrence or major bleeding [50]. Of note, the experience with LMWH has shown good patient adherence with self-injection [49-51, 53]. 
Further, it should be noted in comparison to unfractionated heparin, LMWH reduces the risk of recurrent thrombosis by $32 \%$, major bleeding by $43 \%$, and death by $24 \%$ [54]. Thus, in considering the aforementioned observations taken collectively, LMWH can be considered a preferred agent for treatment of cancer-associated VTE because of its efficacy, safety, and convenience.

One special case for VTE treatment with LMWH, which deserves special consideration, relates to patients with high grade gliomas, for example, glioblastoma multiforme (GBM). VTE occurs in $20-30 \%$ of patients with malignant glioma per year of survival $[55,56]$. These patients are also at high risk for bleeding with and without anticoagulation. In one study discussed in detail below (Section 3, Glioma), the incidence of bleeding was 5.1\% [57]. In a review on the subject, Chao and Robins suggested treating VTE in patients with GBM with full dose LMWH until resolution of symptoms from their thrombosis (usually within 2 weeks of onset of VTE), followed by $50 \%$ dose reduction in the following month, and followed by another $25 \%$ dose reduction after 2 to 3 months [58]. They further recommended dividing the total daily dose during the acute phase of therapy into twicea-day or split dosing to reduce bleeding risk. Parenthetically, one study demonstrated split dosing of dalteparin was more efficacious [59]. Two other studies, one with enoxaparin [60], and one with dalteparin [61], suggested a trend, which was not statistically significant, for better efficacy with split dosing. These authors estimated the incidence of recurrent VTE at less than 5\%. Should any patient develop recurrent VTE during dose reduction of $\mathrm{LMWH}$, it was suggested to check the anti-Xa factor levels (as appropriate to the type of LMWH used) after an injection and adjust the dose accordingly $[58,61,62]$. The aforementioned treatment algorithm (which had been extended to other tumor types, e.g., breast cancer patients) was based on experience. The use of a lower dose of LMWH after acute treatment was felt consistent with the efficacy of LMWH in the prophylaxis setting (see also Section 3) and had evolved as a conceptual extrapolation. It has been the practice of many neuro-oncologists to use LMWH indefinitely in high grade glioma patients. This is consistent with the experience in a Eastern Cooperative Oncology Group (ECOG)/Radiation Therapy Oncology Group (RTOG) trial, described in detail in Section 3 below [63].

2.3. Other Adverse Side Effects. It is worth noting that patients receiving LMWH for extended periods are at increased risk for osteoporosis, and may have other risk factors for bone demineralization, such as the concurrent use of steroids, hence, monitoring these patients with bone mineral density studies, and considering interventions (e.g., bisphosphonates) as appropriate.

2.4. Alternatives to Low-Molecular-Weight Heparin and Warfarin in Treatment of VTE. Fondaparinux, a synthetic selective inhibitor of activated factor X (with no activity against thrombin), is approved for the initial therapy of VTE [64]. In terms of cost and administration convenience, it is comparable to LMWH. Fondaparinux, however, has not been studied specifically in cancer populations. As a result, its use in cancer patients has generally been limited in patients who have a history of heparin-induced thrombocytopenia or allergies to heparin [65].

The use of vena cava filters, as part of treatment of VTE in cancer patients, is not well defined, as previously reviewed [65]. As is obvious, filters do not change the underlying hypercoagulable state in cancer patients. The use of filters in patients receiving anticoagulation has been observed to reduce the incidence of symptomatic pulmonary embolism, but also resulted in more recurrent DVTs and no difference in overall survival [66]. Thus, many clinicians believe filters should be reserved for situations in which anticoagulant therapy is contraindicated, for example, severe thrombocytopenia, active bleeding, or in anticipation of a significant neurosurgical intervention.

\section{Low-Molecular-Weight Heparin as Prophylaxis for Venous Thromboembolism}

Cancer patients are at high risk of VTE, especially in the setting of immobility, hospitalization, or surgery [13, 14, 16, 67]. However, randomized clinical trials of VTE prophylaxis during hospitalization have been conducted in general medical and surgical patients only. There has not been a randomized trial in VTE prophylaxis targeting a hospitalized medical (as opposed to surgical) cancer patient population. There have been three large, randomized, double blind, placebo-controlled trials demonstrating the benefits of anticoagulant prophylaxis, with LMWH or fondaparinux, in general medical patients [68-70]. More than 1,000 hospitalized patients with either congestive heart failure, acute respiratory failure without requiring ventilator support, acute infection, or acute rheumatic disorder in association with an additional risk factor including cancer, were randomly assigned to receive enoxaparin $40 \mathrm{mg}$, enoxaparin $20 \mathrm{mg}$, or placebo subcutaneously once daily for 6 to 14 days; in this study, $12.4 \%$ of patients had cancer [70]. The incidence of VTE was significantly lower in the group that received enoxaparin $40 \mathrm{mg}(5.5 \%)$ than in the group that received placebo (14.9\%; RR $=0.37 ; P<.001)$. There was no significant difference between the groups that received $20 \mathrm{mg}$ of enoxaparin and placebo, respectively [70]. Similarly, the incidence of VTE in patients hospitalized with an acute medical condition was reduced from $4.96 \%$ in the group that received placebo to $2.77 \%$ in the group that received dalteparin 5,000 units subcutaneously once daily, representing a relative risk reduction of $45 \%$ (95\% CI, $38 \%$ to $80 \%$; $P=.0015$ ) [69]. A subgroup analysis from the first study showed that cancer patients had an elevated risk of VTE and that prophylaxis with $40 \mathrm{mg}$ of enoxaparin daily in cancer patients led to a relative risk reduction of 0.5 (95\% CI, 0.14 to 1.72 ), which was similar to the benefits observed in the whole group, despite being underpowered and not reaching statistical significance [71]. It can be inferred from these studies that VTE prophylaxis with LMWH in hospitalized cancer patients would be beneficial. 
For any given site of operation, patients with malignancy undergoing surgery have a higher reported frequency of VTE in the first 90 days after operation [72]. In a multicenter, randomized, and double-blind study, 332 patients undergoing open curative surgery for abdominal or pelvic cancer received one of two durations of postoperative prophylaxis for VTE with enoxaparin $40 \mathrm{mg}$ once daily, either for 28 days postoperatively, or for 6 to 10 days after operation until hospital discharge followed by placebo injections for a further 21 days [73]. The frequency of VTE decreased from $12 \%$ in the placebo group to $4.8 \%$ in the treatment arm $(P=$ .02 ), based on screening venography at the end of the 28day period [73]. The benefits of extended VTE prophylaxis were maintained for 3 months after operation without any evidence of a rebound thrombosis [73].

The role of VTE prevention in ambulatory patients with cancer is controversial. In a randomized, placebo-controlled European trial, 353 patients with metastatic breast cancer received either certoparin at 3,000 anti-Factor Xa units daily or placebo for 6 months; the rate of VTE in each treatment group was $4 \%$ [74]. In a similarly designed trial, the rate of VTE in 547 patients with stage III or stage IV nonsmallcell lung cancer was $4.5 \%$ in the LMWH arm compared with $8.3 \%$ in patients receiving placebo $(P=.07)$ [74]. In a subgroup analysis involving patients with stage IV lung cancer, LMWH led to a statistically significant reduction in VTE $(10.1 \%$ versus $3.5 \%$; $P=.03)$. Certoparin is not currently available in the United States.

The largest ambulatory study to date on VTE prophylaxis was concluded by Agnelli and colleagues [52]. This study addressed the clinical benefit of the LMWH, that is, nadroparin, for the prophylaxis of thromboembolic events in ambulatory patients receiving chemotherapy for metastatic or locally advanced solid cancer. The primary study endpoint was the incidence of symptomatic venous or arterial thromboembolic events. Patients $(n=1150)$ were randomized $(2: 1)$ between nadroparin and placebo group. Results demonstrated $15(2.0 \%)$ of 769 patients treated with nadroparin and $15(3.9 \%)$ of 381 patients treated with placebo had a thromboembolic event (single-sided $P=.02$ ). Five $(0.7 \%)$ of 769 patients in the nadroparin group, and no patients in the placebo group, had a major bleeding event (two-sided $P=.18$ ). The incidences of minor bleeding were $7.4 \%$ ( 57 of 769 ) with nadroparin and $7.9 \%$ (30 of 381) with placebo. There were $121(15.7 \%)$ serious adverse events in the nadroparin group and $67(17.6 \%)$ serious adverse events in the placebo group.

The CONKO 004 trial addressed chemotherapy with or without LMWH, that is, enoxaparin $(1 \mathrm{mg} / \mathrm{kg})$ in patients $(n=312)$ with advanced pancreatic cancer [75]. The primary endpoint was the reduction of symptomatic VTE within the first 12 weeks of treatment. Secondary endpoints were toxicity, time to progression, and overall survival. There were 22 VTE in 152 patients in the observation group and 8 in 160 in the LMWH group. Per-protocol (PP) and intent to treat (ITT) analyses demonstrated significant risk reductions from $14.5 \%$ to $5.0 \%$ (65\% RRR) and $14.5 \%$ to $3.8 \%$ (74\% RRR) for LMWH, respectively. Similarly, the UK FRAGEM study reported a $62 \%$ risk reduction in
VTE using the CLOT therapeutic regimen of dalteparin (31\% versus $12 \% ; P=.02$ ) [76]. Major bleeding events were $9.9 \%$ for the observation group and $6.3 \%$ for LMWH (ITT). In each group there was one tumor-related fatal hemorrhage. Preliminary data demonstrated no difference in time to progression, or survival for observation versus LMWH, respectively, (19 versus 22 weeks) and (29 versus 31 weeks) [75].

\subsection{Special Considerations for Low-Molecular-Weight Heparin Prophylaxis}

3.1.1. Neurosurgery. Prophylaxis in neurosurgical patients has been studied as a unique patient cohort. Two multicenter, randomized, double-blind trials studied the use of LMWH (nadroparin 7500 units subcutaneously once daily, or enoxaparin $40 \mathrm{mg}$ subcutaneously once daily, resp.) versus placebo in conjunction with the use of compression stockings in the prevention of VTE after elective neurosurgery $[77,78]$. The majority of the accrued patients had brain tumors, up to $30 \%$ of whom were diagnosed with glioma [78]. LMWH or placebo was given within 24 hours after surgery and continued for up to 10 days or until hospital discharge. Both studies showed that LMWH combined with compression stockings was more effective than compression stockings alone for the prevention of VTE after elective neurosurgery, without inducing any significant increase of major bleeding. In the nadroparin study [77] there was a trend for a relative risk reduction in DVT of $28 \%$ with a $1.5 \%$ increase in nonfatal bleeding. The enoxaparin study [78] demonstrated statistically significant relative risk of 0.52 and no difference in major bleeding risk. However, it should be noted that LMWH in these studies was given within 24 hours after surgery. Another randomized study conducted at the University of Michigan, where LMWH (enoxaparin $30 \mathrm{mg}$ subcutaneously every 12 hours) was initiated before the induction of anesthesia and was continued throughout the hospital stay, showed that enoxaparin therapy initiated at the time of anesthesia induction significantly increased postoperative intracranial hemorrhage [79]. This study was terminated early because of the increased incidence of adverse events in the enoxaparin treatment group. In conclusion, LMWH given within 24 hours after neurosurgery, but not prior to neurosurgery, in combination with compression stockings, can be considered for the prevention of VTE in glioma patients when continued for up to 10 days.

3.1.2. Glioma. As alluded to above, glioma patients are highly predisposed to thromboembolic phenomena with an incidence approaching $\sim 30 \%[55,56]$. In a phase II study, Robins and colleagues studied patients with newly diagnosed GBM: this patient group received dalteparin at 5,000 units daily during and after radiation [63]. The primary endpoint of the study was survival; the secondary end point was the incidence of VTE. This clinical trail closed prematurely $(n=42)$, as standard of care for GBM changed with the introduction of temozolomide [80]. As the study was originally conceived it was estimated that 
$\sim 30 \%$ of the enrolled patients would develop VTE. Based on statistical estimates, a study cohort of 72 patients was planned, resulting in $81 \%$ power estimate to detect a decrease from $30 \%$ to $15 \%$ in VTE. When the study was concluded there were no observed VTE in patients actively receiving dalteparin. Time on dalteparin ranged from 1.2 months to 25.4 months, with a median time of 6.3 months. There were no reports of grade $3 / 4$ bleeding or thrombocytopenia related to dalteparin before or aftert progression. There were local site reactions to dalteparin ( $\sim 71 \%$ of patients); 2 patients experienced grade 3 toxicities ascribed to radiation. Two patients developed DVT after stopping dalteparin, at a time when they had developed progressive GBM.

A seemingly contradictory result, reported by Perry and colleagues, relates to a phase III Canadian study in which 186 malignant glioma patients were randomized to dalteparin at 5,000 units daily versus placebo. Unfortunately, this trial also closed prematurely, due to lack of drug availability. It was planned to enroll 512 patients. In this study the primary endpoint was the incidence of VTE. There was a trend for a decrease in VTE; however, it was not statistically significant $(\mathrm{HR}=0.51,95 \% \mathrm{CI}: 0.19-1.4, P=.29)$. There was also a trend for increased bleeding in the LMWH arm, that is, 5.1\% $(n=5)$ versus $1.2 \%(n=1)$ for the placebo. The clinical significance of this finding is uncertain, as intratumoral hemorrhage is often part of the natural history of this disease [57]. Noteworthy differences in these studies may relate to differences in patient populations studied. The RTOG study was restricted to GBM patients (as opposed to all malignant glioma); there was also a requirement for an unusually good performance status, that is, 95\% ECOG performance status of 0 or 1 [57].

The results of these two studies, taken collectively, leave the role of thromboembolic prophylaxis with an anticoagulant as undefined for this group of patients with central nervous system disease. As a result, further investigation is required to reach definitive conclusions regarding the application of VTE prophylaxis beyond the postoperative period after hospital.

\section{Coagulation as a Host Factor in Cancer}

The association of disseminated intravascular coagulopathy (DIC) and cancer is well documented in various malignancies, including melanoma, breast, colon, gall bladder, ovary, prostate, and stomach cancers [81]. This association can be explained by general factors related to the host's response to cancer and, more specifically, to factors related to procoagulant activities expressed by the cancer cells [82]. Thrombosis occurs in 5-15\% of patients with cancer [83], comparing to $0.1 \%$ in the general population [84].

Indeed, the occurrence of venous thrombosis in an otherwise healthy person may be the first sign of an occult malignancy [85-87], as malignancy is often associated with thromboembolic disease [81]. Investigations have shown that fibrin is abundant in the stroma of many tumors [88-90]. The long-observed association of clotting with malignancy prompted scientists over 30 years ago to test the hypothesis that coagulation played an adjunctive role in cancer progression. As reviewed by Zacharski and colleagues, a number of experimental animal studies (and limited number of small-scale clinical trials in cancer patients) suggested that anticoagulants, including heparin and warfarin, might successfully inhibit tumor spread [91]. While a role for LMWHs (and perhaps heparin in general) in cancer treatment can be logically argued based on theories of fibrin and tumor implantation, heparin may have important antitumor effects which are independent of their anticoagulant properties. Folkman and colleagues, for example, found that heparin fragments lacking evident anticoagulant activity could still effectively inhibit angiogenesis in presence of cortisone [92]. Other investigators have reported that, individually, high-molecular-weight fractions of heparin stimulate, while low-molecular-weight fractions inhibit, angiogenesis and that this effect was independent of anticoagulation [93]. Furthermore, Bitan and colleagues have shown that the ability of heparin and heparin fragments to inhibit metastasis of melanomas, by inhibiting heparinasedependent degradation of the extracellular matrix, was the same in both anticoagulant and non-anticoagulant fractions [94]. As cancers are most typically manifested by uncontrolled cellular proliferation, it may also be relevant that both anticoagulant and non-anticoagulant fractions of heparin and heparin fragments can inhibit the proliferation of vascular smooth muscle cells [95]. Collen and colleagues have demonstrated the inhibitory effect of heparins on the proliferation of human microvascular endothelial cells, which provides a novel mechanism by which LMWH may affect tumor progression, namely, reduced ingrowth of microvascular structures in a fibrinous stromal matrix by rendering it less permissive for invasion [96].

\section{Anticoagulation and Cancer Survival}

It is of historical interest to note that in 1976 the Veterans Administration (VA) Cooperative Study Program initiated a prospective randomized clinical trial of warfarin in cancer patients to explicitly test the hypothesis that coagulation was involved in cancer progression [91]. In providing a rationale for their trial, the investigators proposed that clotting may (1) facilitate attachment of metastatic tumor cells to the endothelium; (2) provide growth factors for the tumor; (3) provide a structural lattice for cell proliferation; (4) provide tumor cells protection from host immune defenses [91]. As reported in 1984, the group found no differences in survival between warfarin-treated and control groups for several cancers. However, warfarin therapy was associated with a significant prolongation in the time to first evidence of disease progression $(P=.016)$ and a significant improvement in survival $(P=.018)$ for patients with small cell carcinoma of the lung, including the subgroup of patients with disseminated disease at the time of randomization $(P=$ .013) [97].

Interest in the potential relationship between neoplastic diseases and coagulation followed the publication of reports suggesting improved prognosis in cancer patients with thrombosis receiving low-molecular weight-heparin (LMWH) [98]. A meta-analysis of a number of randomized 
clinical trials suggested the superiority of LMWH over standard heparin in subgroups of patients with cancer [98]. Two early studies from this literature also suggest an unexpected and unexplained benefit of LMWH as compared to unfractionated heparin treatment: an apparent reduction in cancer-related mortality in the first three months following initiation of treatment $[86,99]$. A review of published clinical trials involving $\mathrm{LMWH}$ as treatment and prophylaxis for venous thrombosis analyzed a few other studies with data on cancer-related mortality [100]. Although many LMWH studies have reported patients' baseline characteristics, which sometimes included cancer, the follow-up periods were not of significant duration to allow a meaningful assessment of treatment effect on mortality. A meta-analysis of LMWH versus unfractionated heparin (UFH) studies found the 90day mortality in patients with cancer in four studies to be $14 \%$ in the LMWH group, and $28 \%$ in the heparin group $(P=.01)[86,99-102]$.

It is of interest to note that in the aforementioned CLOT study (in Section 2 above), which was a randomized trial of dalteparin for 6 months compared to dalteparin for a brief initial period followed by a vitamin $\mathrm{K}$ antagonist, survival was assessed as a secondary outcome; the primary outcome was rate of recurrent thrombosis [53]. Survival was not found to be improved in the dalteparin arm compared with the control arm. However, a post hoc subgroup analysis, focusing only on the patients with nonmetastatic disease, reported the 12-month all-cause mortality of 35\% in vitamin $\mathrm{K}$ antagonist treated patients compared to $20 \%$ in the dalteparin group $(P=.04)$. Conversely, in patients with metastatic cancer, no survival difference was observed between dalteparin (72\%) and oral anticoagulant (69\%) study arms $(P=.46)[103]$.

LMWH has also been studied as an antineoplastic agent. A randomized controlled study $(n=84)$ found a 4 month improvement in progression free survival $(P=.01)$ in patients with small cell lung cancer patients receiving combination chemotherapy with LMWH compared to those who did not receive LMWH [104]. In another randomized, placebo-controlled study (The Famous Trial) evaluated 385 patients with advanced malignancy receiving standard treatment with or without dalteparin. Although there was no statistically significant benefit overall in the dalteparin arm, in a post hoc landmark analysis of 102 patients still alive at 17 months, a significant improvement in survival was observed for patients receiving dalteparin [105]. A similar observation was also made in a small study of GBM patients reviewed in Section 3 [63], where better survival was seen in patients maintained on dalteparin compared to those who stopped dalteparin after first disease progression. In another randomized study (MALT) $(n=302)$ using nadroparin, a significant improvement in overall survival was observed (relative risk, $0.75 ; 95 \% \mathrm{CI}, 0.59$ to $0.96 ; P=.02$ ) [106]. However, a similar but smaller phase 3 study $(n=144)$ using dalteparin was a negative trial [107].

The aforementioned laboratory and clinical studies are a sampling of the mounting evidence that implicates components of the clotting cascade and neoplastic tumor progression. An excellent review of the subject has been written by Kuderer and colleagues [34]. We agree with these authors that LMWH and related agents hold promise for improving cancer outcomes.

\section{Conclusions}

VTE is a significant source of morbidity and mortality for patients with cancer. LMWHs are safe and effective in treatment and prophylaxis of VTE. Continued research regarding the antineoplastic activity of LMWH alluded to above may provide valuable insights into cancer pathogenesis and new treatment paradigms. Future controlled clinical trials directed at evaluating antithrombotic agents relative to treatment and prophylaxis are clearly warranted in this highrisk population. Other areas for needed research include markers and defined patients pathways to better define patient cohorts who are most likely to develop VTE.

\section{Acknowledgment}

H. I. Robins has served as a consultant to Eisai, Inc in 2010. This paper was supported Kathleen Reader Memorial Research Fund.

\section{References}

[1] A. A. Shlebak and D. B. Smith, "Incidence of objectively diagnosed thromboembolic disease in cancer patients undergoing cytotoxic chemotherapy and/or hormonal therapy," Cancer Chemotherapy and Pharmacology, vol. 39, no. 5, pp. 462-466, 1997.

[2] A. Y. Y. Lee and M. N. Levine, "Venous thromboembolism and cancer: risks and outcomes," Circulation, vol. 107, no. 23, pp. I17-I21, 2003.

[3] N. Goldenberg, S. R. Kahn, and S. Solymoss, "Markers of coagulation and angiogenesis in cancer-associated venous thromboembolism," Journal of Clinical Oncology, vol. 21, no. 22, pp. 4194-4199, 2003.

[4] M. G. Hamilton, R. D. Hull, and G. F. Pineo, "Venous thromboembolism in neurosurgery and neurology patients: a review," Neurosurgery, vol. 34, no. 2, pp. 280-296, 1994.

[5] T. J. Iberti, M. Miller, A. Abalos et al., "Abnormal coagulation profile in brain tumor patients during surgery," Neurosurgery, vol. 34, no. 3, pp. 389-395, 1994.

[6] P. Millac, "Platelet stickness in patients with intracranial tumours," British Medical Journal, vol. 4, no. 570, pp. 25-26, 1967.

[7] M. Nathanson and J. P. Savitsky, "Platelet adhesive index studies in multiple sclerosis and other neurological disorders," Bulletin of the New York Academy of Medicine, vol. 28, pp. 462-468, 1952.

[8] S. Sallah, A. Husain, V. Sigounas et al., "Plasma coagulation markers in patients with solid tumors and venous thromboembolic disease receiving oral anticoagulation therapy," Clinical Cancer Research, vol. 10, no. 21, pp. 7238-7243, 2004.

[9] G. F. V. Tempelhoff, M. Dietrich, G. Hommel, and L. Heilmann, "Blood coagulation during adjuvant epirubicin/cyclophosphamide chemotherapy in patients with primary operable breast cancer," Journal of Clinical Oncology, vol. 14, no. 9, pp. 2560-2568, 1996. 
[10] G. F. Von Tempelhoff, M. Dietrich, F. Niemann, D. Schneider, G. Hommel, and L. Heilmann, "Blood coagulation and thrombosis in patients with ovarian malignancy," Thrombosis and Haemostasis, vol. 77, no. 3, pp. 456-461, 1997.

[11] J. A. Heit, M. D. Silverstein, D. N. Mohr, T. M. Petterson, W. M. O’Fallon, and L. J. Melton, "Risk factors for deep vein thrombosis and pulmonary embolism: a population-based case-control study," Archives of Internal Medicine, vol. 160, no. 6, pp. 809-815, 2000.

[12] J. W. Blom, C. J. M. Doggen, S. Osanto, and F. R. Rosendaal, "Malignancies, prothrombotic mutations, and the risk of venous thrombosis," Journal of the American Medical Association, vol. 293, no. 6, pp. 715-722, 2005.

[13] P. D. Stein, A. Beemath, F. A. Meyers, E. Skaf, J. Sanchez, and R. E. Olson, "Incidence of venous thromboembolism in patients hospitalized with cancer," American Journal of Medicine, vol. 119, no. 1, pp. 60-68, 2006.

[14] A. A. Khorana, C. W. Francis, E. Culakova, N. M. Kuderer, and G. H. Lyman, "Frequency, risk factors, and trends for venous thromboembolism among hospitalized cancer patients," Cancer, vol. 110, no. 10, pp. 2339-2346, 2007.

[15] H. K. Chew, T. Wun, D. Harvey, H. Zhou, and R. H. White, "Incidence of venous thromboembolism and its effect on survival among patients with common cancers," Archives of Internal Medicine, vol. 166, no. 4, pp. 458-464, 2006.

[16] N. Levitan, A. Dowlati, S. C. Remick et al., "Rates of initial and recurrent thromboembolic disease among patients with malignancy versus those without malignancy: risk analysis using medicare claims data," Medicine, vol. 78, no. 5, pp. 285291, 1999.

[17] J. W. Blom, J. P. M. Vanderschoot, M. J. Oostindiër, S. Osanto, F. J. M. Van Der Meer, and F. R. Rosendaal, "Incidence of venous thrombosis in a large cohort of 66329 cancer patients: results of a record linkage study," Journal of Thrombosis and Haemostasis, vol. 4, no. 3, pp. 529-535, 2006.

[18] A. A. Khorana and G. C. Connolly, "Assessing risk of venous thromboembolism in the patient with cancer," Journal of Clinical Oncology, vol. 27, no. 29, pp. 4839-4847, 2009.

[19] A. A. Khorana, C. W. Francis, E. Culakova, N. M. Kuderer, and G. H. Lyman, "Thromboembolism is a leading cause of death in cancer patients receiving outpatient chemotherapy," Journal of Thrombosis and Haemostasis, vol. 5, no. 3, pp. 632634, 2007.

[20] M. N. Levine, M. Gent, J. Hirsch et al., "The thombogenic effect of anticancer drug therapy in women with Stage II breast cancer," New England Journal of Medicine, vol. 318, no. 7, pp. 404-407, 1988.

[21] H. T. Sørensen, L. Mellemkjær, J. H. Olsen, and J. A. Baron, "Prognosis of cancers associated with venous thromboembolism," New England Journal of Medicine, vol. 343, no. 25, pp. 1846-1850, 2000.

[22] A. Falanga and M. Marchetti, "Venous thromboembolism in the hematologic malignancies," Journal of Clinical Oncology, vol. 27, no. 29, pp. 4848-4857, 2009.

[23] M. Mandalà, M. Reni, S. Cascinu et al., "Venous thromboembolism predicts poor prognosis in irresectable pancreatic cancer patients," Annals of Oncology, vol. 18, no. 10, pp. 1660$1665,2007$.

[24] A. A. Brandes, E. Scelzi, G. Salmistraro et al., "Incidence and risk of thromboembolism during treatment of high-grade gliomas: a prospective study," European Journal of Cancer, vol. 33, no. 10, pp. 1592-1596, 1997.

[25] K. Osman, R. Comenzo, and S. V. Rajkumar, "Deep venous thrombosis and thalidomide therapy for multiple myeloma,"
New England Journal of Medicine, vol. 344, no. 25, pp. 19511952, 2001.

[26] S. V. Rajkumar, E. Blood, D. Vesole, R. Fonseca, and P. R. Greipp, "Phase III clinical trial of thalidomide plus dexamethasone compared with dexamethasone alone in newly diagnosed multiple myeloma: a clinical trial coordinated by the eastern cooperative oncology group," Journal of Clinical Oncology, vol. 24, no. 3, pp. 431-436, 2006.

[27] R. Knight, R. J. DeLap, and J. B. Zeldis, "Lenalidomide and venous thrombosis in multiple myeloma," New England Journal of Medicine, vol. 354, no. 19, pp. 2079-2080, 2006.

[28] J. A. Zonder, B. Barlogie, B. G. M. Durie, J. McCoy, J. Crowley, and M. A. Hussein, "Thrombotic complications in patients with newly diagnosed multiple myeloma treated with lenalidomide and dexamethasone: benefit of aspirin prophylaxis," Blood, vol. 108, no. 1, p. 403, 2006.

[29] A. K. Kakkar and R. C. N. Williamson, "Prevention of venous thromboembolism in cancer using low-molecularweight heparins," Haemostasis, vol. 27, supplement 1, pp. 3237, 1997.

[30] J. L. Ambrus, C. M. Ambrus, I. B. Mink, and J. W. Pickren, "Causes of death in cancer patients," Journal of Medicine, vol. 6, no. 1, pp. 61-64, 1975.

[31] V. Bobek, M. Boubelík, J. Kovarik, and O. Taltynov, "Inhibition of adhesion breast cancer cells by anticoagulant drugs and cimetidine," Neoplasma, vol. 50, no. 2, pp. 148-151, 2003.

[32] V. Bobek and J. Kovarik, "Antitumor and antimetastatic effect of warfarin and heparins," Biomedicine and Pharmacotherapy, vol. 58, no. 4, pp. 213-219, 2004.

[33] M. Hejna, M. Raderer, and C. C. Zielinski, "Inhibition of metastases by anticoagulants," Journal of the National Cancer Institute, vol. 91, no. 1, pp. 22-36, 1999.

[34] N. M. Kuderer, T. L. Ortel, and C. W. Francis, "Impact of venous thromboembolism and anticoagulation on cancer and cancer survival," Journal of Clinical Oncology, vol. 27, no. 29, pp. 4902-4911, 2009.

[35] A. E. Lee, L. A. Rogers, J. M. Longcroft, and R. E. Jeffery, "Reduction of metastasis in a murine mammary tumour model by heparin and polyinosinic-polycytidylic acid," Clinical and Experimental Metastasis, vol. 8, no. 2, pp. 165-171, 1990.

[36] J. Kyo Lee, B. Choi, R. A. Sobel, E. A. Chiocca, and R. L. Martuza, "Inhibition of growth and angiogenesis of human neurofibrosarcoma by heparin and hydrocortisone," Journal of Neurosurgery, vol. 73, no. 3, pp. 429-435, 1990.

[37] R. J. Ludwig, B. Boehme, M. Podda et al., "Endothelial P-selectin as a target of heparin action in experimental melanoma lung metastasis," Cancer Research, vol. 64, no. 8, pp. 2743-2750, 2004.

[38] B. Maat and P. Hilgard, "Anticoagulants and experimental metastases - evaluation of antimetastatic effects in different model systems," Journal of Cancer Research and Clinical Oncology, vol. 101, no. 3, pp. 275-283, 1981.

[39] T. Sciumbata, P. Caretto, P. Pirovano et al., "Treatment with modified heparins inhibits experimental metastasis formation and leads, in some animals, to long-term survival," Invasion and Metastasis, vol. 16, no. 3, pp. 132-143, 1996.

[40] S. M. Smorenburg and C. J. F. Van Noorden, "The complex effects of heparins on cancer progression and metastasis in experimental studies," Pharmacological Reviews, vol. 53, no. 1, pp. 93-105, 2001.

[41] S. M. Smorenburg, R. Vink, M. T. Lintelo et al., "In vivo treatment of rats with unfractionated heparin (UFH) or low 
molecular weight heparin (LMWH) does not affect experimentally induced colon carcinoma metastasis," Clinical and Experimental Metastasis, vol. 17, no. 5, pp. 451-456, 1999.

[42] J. L. Stevenson, S. H. Choi, and A. Varki, "Differential metastasis inhibition by clinically relevant levels of heparinscorrelation with selectin inhibition, not antithrombotic activity," Clinical Cancer Research, vol. 11, no. 19, pp. 70037011, 2005.

[43] E. A. Johnson, T. B. L. Kirkwood, Y. Stirling et al., "Four heparin preparations: anti-Xa potentiating effect of heparin after subcutaneous injection," Thrombosis and Haemostasis, vol. 35, no. 3, pp. 586-591, 1976.

[44] B. Boneu, C. Caranobe, Y. Cadroy et al., "Pharmacokinetic studies of standard unfractionated heparin, and low molecular weight heparins in the rabbit," Seminars in Thrombosis and Hemostasis, vol. 14, no. 1, pp. 18-27, 1988.

[45] M. Palm and C. Mattsson, "Pharmacokinetics of heparin and low molecular weight heparin fragment (fragmin) in rabbits with impaired renal or metabolic clearance," Thrombosis and Haemostasis, vol. 58, no. 3, pp. 932-935, 1987.

[46] H. L. Casele, S. A. Laifer, D. A. Woelkers, and R. Venkataramanan, "Changes in the pharmacokinetics of the lowmolecular-weight heparin enoxaparin sodium during pregnancy," American Journal of Obstetrics and Gynecology, vol. 181, no. 5, pp. 1113-1117, 1999.

[47] R. Abbate, A. M. Gori, A. Farsi, M. Attanasio, and G. Pepe, "Monitoring of low-molecular-weight heparins in cardiovascular disease," American Journal of Cardiology, vol. 82, pp. 33L-36L, 1998.

[48] B. Boneu, "Low molecular weight heparin therapy: is monitoring needed?" Thrombosis and Haemostasis, vol. 72, no. 3, pp. 330-334, 1994.

[49] S. R. Deitcher, C. M. Kessler, G. Merli, J. R. Rigas, R. M. Lyons, and J. Fareed, "Secondary prevention of venous thromboembolic events in patients with active cancer: enoxaparin alone versus initial enoxaparin followed by warfarin for a 180-day period," Clinical and Applied Thrombosis/Hemostasis, vol. 12, no. 4, pp. 389-396, 2006.

[50] G. Meyer, Z. Marjanovic, J. Valcke et al., "Comparison of lowmolecular-weight heparin and warfarin for the secondary prevention of venous thromboembolism in patients with cancer: a randomized controlled study," Archives of Internal Medicine, vol. 162, no. 15, pp. 1729-1735, 2002.

[51] R. D. Hull, G. F. Pineo, R. F. Brant et al., "Long-term lowmolecular-weight heparin versus usual care in proximalvein thrombosis patients with cancer," American Journal of Medicine, vol. 119, no. 12, pp. 1062-1072, 2006.

[52] G. Agnelli, G. Gussoni, C. Bianchini et al., "Nadroparin for the prevention of thromboembolic events in ambulatory patients with metastatic or locally advanced solid cancer receiving chemotherapy: a randomised, placebo-controlled, double-blind study," The Lancet Oncology, vol. 10, no. 10, pp. 943-949, 2009.

[53] A. Y. Y. Lee, M. N. Levine, R. I. Baker et al., "Low-molecularweight heparin versus a coumarin for the prevention of recurrent venous thromboembolism in patients with cancer," New England Journal of Medicine, vol. 349, no. 2, pp. 146-153, 2003.

[54] C. J. van Dongen, A. G. van den Belt, M. H. Prins, and A. W. Lensing, "Fixed dose subcutaneous low molecular weight heparins versus adjusted dose unfractionated heparin for venous thromboembolism," Cochrane Database of Systematic Reviews, no. 4, Article ID CD001100, 2004.
[55] R. Brisman and J. Mendell, "Thromboembolism and brain tumors," Journal of Neurosurgery, vol. 38, no. 3, pp. 337-338, 1973.

[56] M. C. Kayser Gatchalian and K. Kayser, "Thrombosis and intracranial tumors," Journal of Neurology, vol. 209, no. 3, pp. 217-224, 1975.

[57] J. R. Perry, J. A. Julian, N. J. Laperriere et al., "PRODIGE: a randomized placebo-controlled trial of dalteparin lowmolecular-weight heparin thromboprophylaxis in patients with newly diagnosed malignant glioma," Journal of Thrombosis and Haemostasis, vol. 8, no. 9, pp. 1959-1965, 2010.

[58] B. H. Chao and H. I. Robins, "The use of low molecular weight heparin in the treatment and prevention of thromboembolic disease in glioma," in Tumors of the Central Nervous System, M. A. Havat, Ed., Springer, New York, NY, USA, 2010.

[59] H. Partsch, B. Kechavarz, A. Mostbeck, H. Kohn, and C. Lipp, "Frequency of pulmonary embolism in patients who have iliofemoral deep vein thrombosis and are treated with once- or twice-daily low-molecular-weight heparin," Journal of Vascular Surgery, vol. 24, no. 5, pp. 774-782, 1996.

[60] G. Merli, T. E. Spiro, C. G. Olsson et al., "Subcutaneous enoxaparin once or twice daily compared with intravenous unfractionated heparin for treatment venous thromboembolic disease," Annals of Internal Medicine, vol. 134, no. 3, pp. 191-202, 2001.

[61] B. A. Duplaga, C. W. Rivers, and E. Nutescu, "Dosing and monitoring of low-molecular-weight heparins in special populations," Pharmacotherapy, vol. 21, no. 2, pp. 218-234, 2001.

[62] J. Hirsh, K. A. Bauer, M. B. Donati, M. Gould, M. M. Samama, and J. I. Weitz, "Parenteral anticoagulants: American College of Chest Physicians evidence-based clinical practice guidelines (8th edition)," Chest, vol. 133, no. 6, pp. 141S-159S, 2008.

[63] H. I. Robins, A. O’Neill, M. Gilbert et al., "Effect of dalteparin and radiation on survival and thromboembolic events in glioblastoma multiforme: a phase II ECOG trial," Cancer Chemotherapy and Pharmacology, vol. 62, no. 2, pp. 227-233, 2008.

[64] H. R. Büller, B. L. Davidson, H. Decousus et al., "Fondaparinux or enoxaparin for the initial treatment of symptomatic deep vienous thrombosis: a randomized trial," Annals of Internal Medicine, vol. 140, no. 11, pp. 867-873, 2004.

[65] A. Y. Y. Lee, "Anticoagulation in the treatment of established venous thromboembolism in patients with cancer," Journal of Clinical Oncology, vol. 27, no. 29, pp. 4895-4901, 2009.

[66] Investigators PdRdEPpIC, "Eight-year follow-up of patients with permanent vena cava filters in the prevention of pulmonary embolism: the PREPIC (Prevention du Risque d'Embolie Pulmonaire par Interruption Cave) randomized study," Circulation, vol. 112, no. 3, pp. 416-422, 2005.

[67] S. Sallah, J. Y. Wan, and N. P. Nguyen, "Venous thrombosis in patients with solid tumors: determination of frequency and characteristics," Thrombosis and Haemostasis, vol. 87, no. 4, pp. 575-579, 2002.

[68] A. T. Cohen, B. L. Davidson, A. S. Gallus et al., "Efficacy and safety of fondaparinux for the prevention of venous thromboembolism in older acute medical patients: randomised placebo controlled trial," British Medical Journal, vol. 332, no. 7537, pp. 325-327, 2006.

[69] A. Leizorovicz, A. T. Cohen, A. G. G. Turpie, C. G. Olsson, P. T. Vaitkus, and S. Z. Goldhaber, "Randomized, 
placebo-controlled trial of dalteparin for the prevention of venous thromboembolism in acutely ill medical patients," Circulation, vol. 110, no. 7, pp. 874-879, 2004.

[70] M. M. Samama, A. T. Cohen, J.-Y. Darmon et al., "A comparison of enoxaparin with placebo for the prevention of venous thromboembolism in acutely ill medical patients," New England Journal of Medicine, vol. 341, no. 11, pp. 793$800,1999$.

[71] R. Alikhan, A. T. Cohen, S. Combe et al., "Risk factors for venous thromboembolism in hospitalized patients with acute medical illness: analysis of the MEDENOX Study," Archives of Internal Medicine, vol. 164, no. 9, pp. 963-968, 2004.

[72] R. H. White, H. Zhou, and P. S. Romano, "Incidence of symptomatic venous thromboembolism after different elective or urgent surgical procedures," Thrombosis and Haemostasis, vol. 90, no. 3, pp. 446-455, 2003.

[73] D. Bergqvist, G. Agnelli, A. T. Cohen et al., "Duration of prophylaxis against venous thromboembolism with enoxaparin after surgery for cancer," New England Journal of Medicine, vol. 346, no. 13, pp. 975-980, 2002.

[74] S. Haas, A. Kakkar, and B. Kemkes-Matthes, "Prevention of venous thromboembolism with low-molecular-weight heparin in patients with metastatic breast or lung cancer: results of the TOPIC studies," Journal of Thrombosis and Haemostasis, vol. 3, article OR059, 2005.

[75] H. Riess, U. Pelzer, G. Deutschinoff et al., "A prospective, randomized trial of chemotherapy with or without the low molecular weight heparin (LMWH) enoxaparin in patients with advanced pancreatic cancer: results of the CONKO 004 trial," Journal of Clinical Oncology, vol. 27, article LBA4506, 2009.

[76] A. Maraveyas, J. Waters, R. Roy et al., "Gemcitabine with or without prophylactic weight-adjusted dalteparin in patients with advanced or metastatic pancreatic cancer (APC): a multicentre, randomised phase IIB trial (the UK FRAGEM study)," European Journal of Cancer, Supplement, vol. 7, article 362, 2009.

[77] M. T. Nurmohamed, A. M. Van Riel, C. M. A. Henkens et al., "Low molecular weight heparin and compression stockings in the prevention of venous thromboembolism in neurosurgery," Thrombosis and Haemostasis, vol. 75, no. 2, pp. 233-238, 1996.

[78] G. Agnelli, F. Piovella, P. Buoncristiani et al., "Enoxaparin plus compression stockings compared with compression stockings alone in the prevention of venous thromboembolism after elective neurosurgery," New England Journal of Medicine, vol. 339, no. 2, pp. 80-85, 1998.

[79] L. D. Dickinson, L. D. Miller, C. P. Patel, and S. K. Gupta, "Enoxaparin increases the incidence of postoperative intracranial hemorrhage when initiated preoperatively for deep venous thrombosis prophylaxis in patients with brain tumors," Neurosurgery, vol. 43, no. 5, pp. 1074-1079, 1998.

[80] R. Stupp, W. P. Mason, M. J. Van Den Bent et al., "Conclusive results of a randomized phase III trial by the EROTC Brain and RT groups and NICIC Clinical Trails Group. Concomitant and adjuvant temozolomide and radiotherapy for newly diagnosed glioblastoma multiforme," Proceedings of the American Society of Clinical Oncology, vol. 23, abstract 2, 2004.

[81] L. V. M. Rao, “Tissue factor as a tumor procoagulant," Cancer and Metastasis Reviews, vol. 11, no. 3-4, pp. 249-266, 1992.

[82] P. M. Mannucci, "Markers of hypercoagulability in cancer patients," Haemostasis, vol. 27, supplement 1, pp. 25-31, 1997.
[83] A. I. Schafer, "The hypercoagulable states," Annals of Internal Medicine, vol. 102, no. 6, pp. 814-828, 1985.

[84] V. A. Drill, "Oral contraceptives and thromboembolic disease," Journal of the American Medical Association, vol. 221, no. 6, pp. 602-603, 1972.

[85] F. R. Rickles, W. W. Hancock, R. L. Edwards, and L. R. Zacharski, "Antimetastatic agents. I. Role of cellular procoagulants in the pathogenesis of fibrin deposition in cancer and the use of anticoagulants and/or antiplatelet drugs in cancer treatment," Seminars in Thrombosis and Hemostasis, vol. 14, no. 1, pp. 88-94, 1988.

[86] P. Prandoni, A. W. A. Lensing, H. R. Buller et al., "Comparison of subcutaneous low-molecular-weight heparin with intravenous standard heparin in proximal deep-vein thrombosis," Lancet, vol. 339, no. 8791, pp. 441-445, 1992.

[87] M. Monreal, J. Fernandez-Llamazares, J. Perandreu, A. Urrutia, J. C. Sahuquillo, and E. Contel, "Occult cancer in patients with venous thromboembolism: which patients, which cancers," Thrombosis and Haemostasis, vol. 78, no. 5, pp. 1316-1318, 1997.

[88] J. Contrino, G. Hair, D. L. Kreutzer, and F. R. Rickles, "In situ detection of tissue factor in vascular endothelial cells: correlation with the malignant phenotype of human breast disease," Nature Medicine, vol. 2, no. 2, pp. 209-215, 1996.

[89] V. Costantini and L. R. Zacharski, "The role of fibrin in tumor metastasis," Cancer and Metastasis Reviews, vol. 11, no. 3-4, pp. 283-290, 1992.

[90] H. F. Dvorak, D. R. Senger, and A. M. Dvorak, "Fibrin as a component of the tumor stroma: origins and biological significance," Cancer and Metastasis Reviews, vol. 2, no. 1, pp. 41-73, 1983.

[91] L. R. Zacharski, W. G. Henderson, and F. R. Rickles, "Rationale and experimental design for the VA cooperative study of anticoagulation (warfarin) in the treatment of cancer," Cancer, vol. 44, no. 2, pp. 732-741, 1979.

[92] J. Folkman, R. Langer, and R. J. Linhardt, "Angiogenesis inhibition and tumor regression caused by heparin or a heparin fragment in the presence of cortisone," Science, vol. 221, no. 4612, pp. 719-725, 1983.

[93] K. Norrby, "Heparin and angiogenesis: a low-molecularweight fraction inhibits and a high-molecular-weight fraction stimulates angiogenesis systemically," Haemostasis, vol. 23, supplement 1, pp. 141-149, 1993.

[94] M. Bitan, M. Mohsen, E. Levi et al., "Structural requirements for inhibition of melanoma lung colonization by heparanase inhibiting species of heparin," Israel Journal of Medical Sciences, vol. 31, no. 2-3, pp. 106-118, 1995.

[95] J. J. Castellot Jr., J. Choay, and J. C. Lormeau, "Structural determinants of the capacity of heparin to inhibit the proliferation of vascular smooth muscle cells. II. Evidence for a pentasaccharide sequence that contains a 3-O-sulfate group," Journal of Cell Biology, vol. 102, no. 5, pp. 1979-1984, 1986.

[96] A. Collen, S. M. Smorenburg, E. Peters et al., "Unfractionated and low molecular weight heparin affect fibrin structure and angiogenesis in Vitro," Cancer Research, vol. 60, no. 21, pp. 6196-6200, 2000.

[97] L. R. Zacharski, W. G. Henderson, and F. R. Rickles, "Effect of warfarin anticoagulation on survival in carcinoma of the lung, colon, head and neck, and prostate. Final report of VA Cooperative Study \#75," Cancer, vol. 53, no. 10, pp. 20462052, 1984. 
[98] A. W. A. Lensing, M. H. Prins, B. L. Davidson, and J. Hirsh, "Treatment of deep venous thrombosis with lowmolecular-weight heparins: a meta-analysis," Archives of Internal Medicine, vol. 155, no. 6, pp. 601-607, 1995.

[99] R. D. Hull, G. E. Raskob, G. F. Pineo et al., "Subcutaneous low-molecular-weight heparin compared with continuous intravenous heparin in the treatment of proximal-vein thrombosis," New England Journal of Medicine, vol. 326, no. 15, pp. 975-982, 1992.

[100] S. Siragusa, B. Cosmi, F. Piovella, J. Hirsh, and J. S. Ginsberg, "Low-molecular-weight heparins and unfractionated heparin in the treatment of patients with acute venous thromboembolism: results of a meta-analysis," American Journal of Medicine, vol. 100, no. 3, pp. 269-277, 1996.

[101] Study CEM, "A randomised trial of subcutaneous low molecular weight heparin (CY 216) compared with intravenous unfractionated heparin in the treatment of deep vein thrombosis. A collaborative European multicentre study," Journal of Thrombosis and Haemostasis, vol. 65, pp. 251-256, 1991.

[102] G. Simonneau, B. Charbonnier, H. Decousus et al., "Subcutaneous low-molecular-weight heparin compared with continuous intravenous unfractionated heparin in the treatment of proximal deep vein thrombosis," Archives of Internal Medicine, vol. 153, no. 13, pp. 1541-1546, 1993.

[103] A. Y. Y. Lee, F. R. Rickles, J. A. Julian et al., "Randomized comparison of low molecular weight heparin and coumarin derivatives on the survival of patients with cancer and venous thromboembolism," Journal of Clinical Oncology, vol. 23, no. 10, pp. 2123-2129, 2005.

[104] M. Altinbas, H. S. Coskun, O. Er et al., "A randomized clinical trial of combination chemotherapy with and without lowmolecular-weight heparin in small cell lung cancer," Journal of Thrombosis and Haemostasis, vol. 2, no. 8, pp. 1266-1271, 2004.

[105] A. K. Kakkar, M. N. Levine, Z. Kadziola et al., "Low molecular weight heparin, therapy with dalteparin, and survival in advanced cancer: the fragmin advanced malignancy outcome study (FAMOUS)," Journal of Clinical Oncology, vol. 22, no. 10, pp. 1944-1948, 2004.

[106] C. P. W. Klerk, S. M. Smorenburg, H. M. Otten et al., "The effect of low molecular weight heparin on survival in patients with advanced malignancy," Journal of Clinical Oncology, vol. 23, no. 10, pp. 2130-2135, 2005.

[107] K. Sideras, P. L. Schaefer, S. H. Okuno et al., "Low-molecularweight heparin in patients with advanced cancer: a phase 3 clinical trial," Mayo Clinic Proceedings, vol. 81, no. 6, pp. 758767, 2006. 


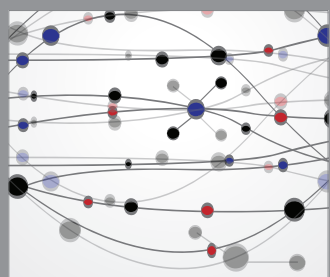

The Scientific World Journal
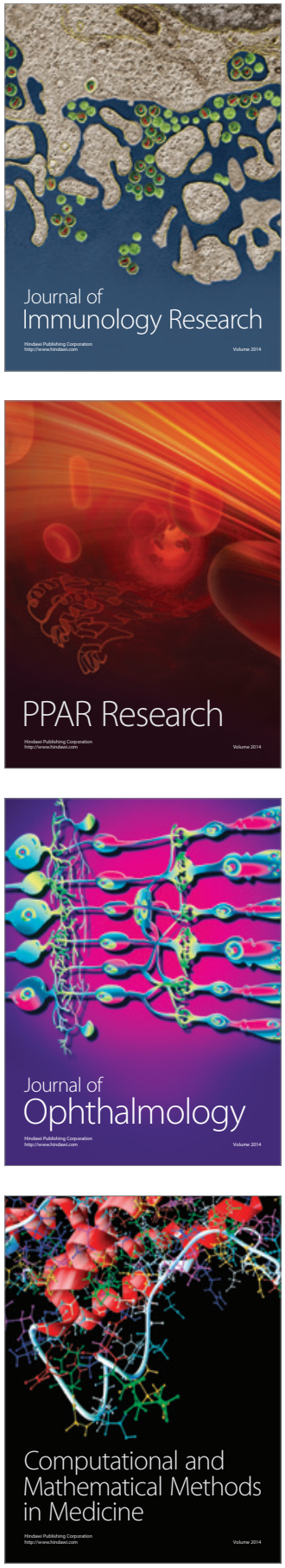

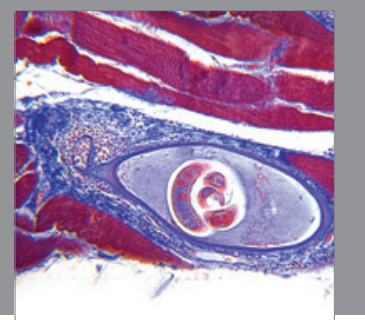

Gastroenterology

Research and Practice
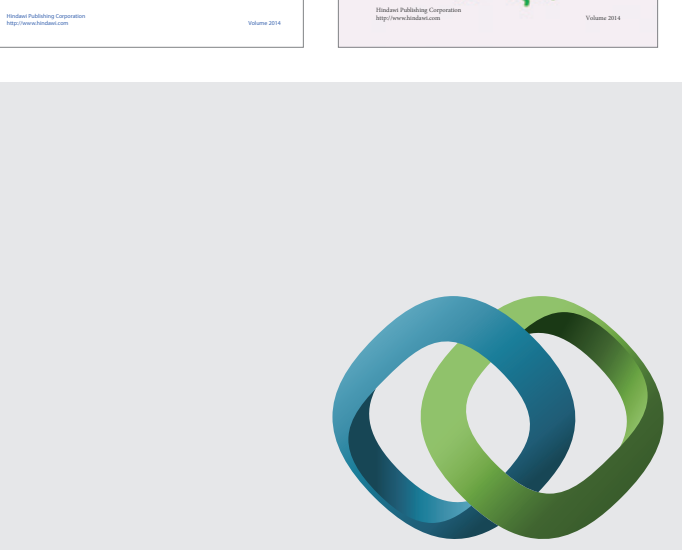

\section{Hindawi}

Submit your manuscripts at

http://www.hindawi.com
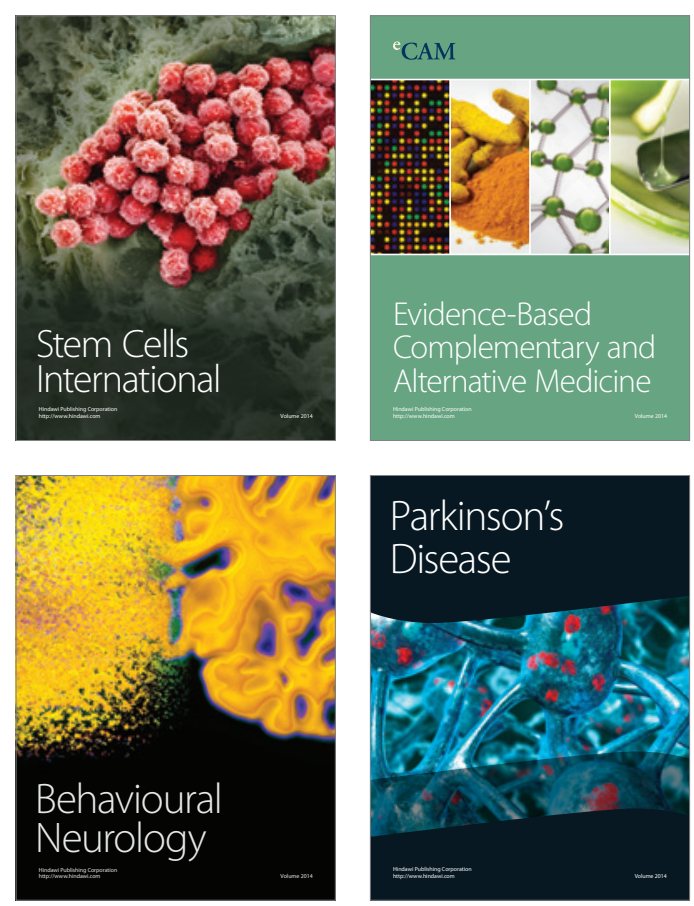

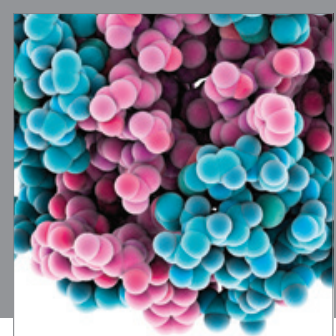

Journal of
Diabetes Research

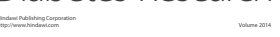

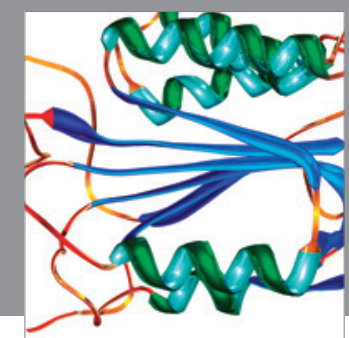

Disease Markers
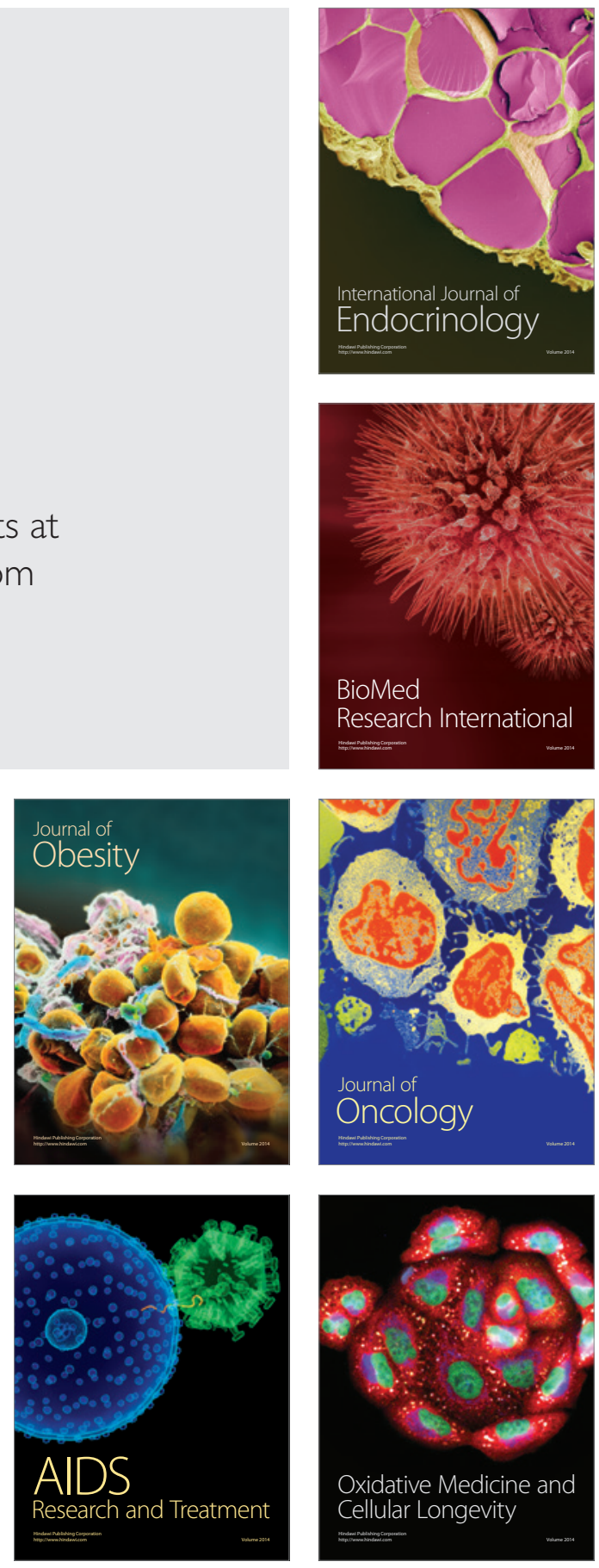